\title{
Causas de hiperglucemia en pacientes internados
}

\section{Causes of hyperglycemia in hospitalized patients}

\author{
Agustina Bonamico $^{1}$ (D), Silvina Dominguez Matheu ${ }^{2}$, Ignacio Gutiérrez Magaldi ${ }^{1}$, Sofía Furrer ${ }^{1}$, Sofía \\ Laura Bianchi ${ }^{1}$. \\ 1.Universidad Católica de Córdoba, Facultad de Ciencias de la Salud, Clínica Universitaria Reina Fabiola, Servicio de Medicina Interna. \\ 2. Universidad Católica de Córdoba, Facultad de Ciencias de la Salud, Clínica Universitaria Reina Fabiola, Servicio de Diabetes. \\ Correspondencia: Agustina Bonamico, email: agusbonamico@gmail.com.
}

\section{Resumen}

INTRODUCCION: La hiperglucemia constituye un factor pronóstico de morbi-mortalidad. Los pacientes con hiperglucemia tienen mayor riesgo de complicaciones intrahospitalarias tales como neumonía, infección de vías urinarias, enfermedad renal aguda, sepsis, muerte durante la internación o en cortos períodos post alta. Durante la internación se recomienda valores de glucemia entre 140-180 mg/dL.

OBJETIVO: Establecer las causas de hiperglucemia en pacientes adultos internados en la Clínica Universitaria Reina Fabiola.

MATERIALES Y METODOS: Estudio observacional, retrospectivo y descriptivo. La población incluyó pacientes mayores de 18 años de ambos sexos que ingresaron al internado del Servicio de Clínica Médica de la Clínica Universitaria Reina Fabiola. Se evaluó la glucemia plasmática al ingreso y a las 72 horas de internación, así como la hemoglobina glicosilada (HbA1c) a los pacientes hiperglucémicos que no tenían diagnóstico de diabetes previo al ingreso hospitalario. Se consideró hiperglucemia a valores de glucemia $\geq 140 \mathrm{mg} / \mathrm{dl}$, se infirió diagnóstico de diabetes mellitus al momento de la internación a la presencia de una HbA1c $\geq 6.5 \%$ y pacientes con hiperglucemia de estrés a los que tenían HbA1c menor a $6.5 \%$ y alguna causa que lo justifique. Variables analizadas: valor de glucemia, hemoglobina glicosilada, edad en años, sexo, antecedente personales patológicos, incluido diabetes mellitus, motivo de internación, situaciones relacionadas a la internación. Análisis estadístico: los datos se presentaron con estadística descriptiva.

RESULTADOS: La muestra final estuvo conformada por un total de $n=900$ pacientes, el 54\% fueron de sexo masculino. La edad media fue de 57,3 años. De los pacientes ingresados, 192 (21.3\%) tenían un valor de glucemia mayor o igual a $140 \mathrm{mg} / \mathrm{dl}$, y a las 72 horas este indicador se elevó a 486 (54\%) pacientes. Con respecto al sexo, no se hallaron diferencias en las mediciones de la glucosa. La hiperglucemia se presentó en mayor proporción en aquellos pacientes que tuvieron una internación no programada: 156 (81\%) vs. 42 (19\%). Del total de pacientes, hubo $126(14 \%)$ con antecedente previo de diabetes. Se les midió la hemoglobina glicosilada a $426(47 \%)$ pacientes que habían presentado hiperglucemia al ingreso o a las 72 hs, $72(16.9 \%)$ presentaron una hemoglobina glicosilada $>6.5 \%$, a los cuales se los podría definir como pacientes con diabetes mellitus de reciente diagnóstico. Presentaron hemoglobina glicosilada $<6.5 \% 342$ $(80.2 \%)$ pacientes que se podrían interpretar clínicamente como el grupo de pacientes con hiperglucemia de estrés. Este grupo tenía las siguientes características: estado postquirúrgico, tratamiento con corticoides, infección activa, terapia inmunosupresora, insuficiencia renal aguda, y nutrición parenteral o alimentación enteral. La hipertensión arterial fue el antecedente personal que se presentó con mayor frecuencia.

CONCLUSION: En este estudio, el $20 \%$ de los pacientes adultos internados presentaron hiperglucemia; este porcentaje coincide con la literatura publicada. Valores de glucemia mayores a $140 \mathrm{mg} / \mathrm{dl}$ estuvieron presentes en pacientes con y sin antecedentes de diabetes mellitus, especialmente en aquellos que cursaron internación por cirugías e infecciones, y en los que estaban con tratamiento con corticoides. También hubo

Revista Methodo: Investigación Aplicada a las Ciencias Biológicas. Universidad Católica de Córdoba Jacinto Ríos 571 Bo Gral. Paz. X5004FXS. Córdoba. Argentina. Tel.: (54) 3514517299 / Correo: methodo@ucc.edu.ar Neb: methodo.ucc.edu.ar | ARTICULO ORIGINAL Rev. Methodo 2022;7(1):31-36. 
pacientes en los que con el hallazgo de hiperglucemia se pudo inferir el diagnóstico de diabetes durante la internación.

Palabras claves: hiperglucemia en internado, hiperglucemia de estrés.

\section{Abstract}

INTRODUCTION: Hyperglycemia is a prognostic factor for mortality. Patients with hyperglycemia have an increased risk of intra-hospital complications, such as pneumonia, urinary tract infection, acute kidney disease, sepsis. During hospitalization, blood glucose values between 140-180 mg / dL are recommended. OBJETIVE: To determine the causes of hyperglycemia in patients hospitalized at Clinica Universitaria Reina Fabiola.

MATERIAL AND METHOD: This is an observational, retrospective and descriptive study. The population included patients over 18 years of both sexes who were admitted to the Medical Clinic Service of the Clinica Universitaria Reina Fabiola. Glycemia at admission and 72 hours after admission as well as glycated hemoglobin $(\mathrm{HbA1c})$ were evaluated in hyperglycemic patients who were not diagnosed with diabetes prior to hospital admission. Hyperglycemia will be considered at blood glucose values greater than $140 \mathrm{mg} / \mathrm{dl}$, diabetes mellitus diagnosed at the time of hospitalization in the presence of an $\mathrm{HbA} 1 \mathrm{c}>6.5 \%$, and patients with stress hyperglycemia who have HbAlc less than $6.5 \%$ and some cause that justifies it. Variables to be analyzed: glycemic value, glycated hemoglobin, age in years, sex, personal pathological history, reason for hospitalization, situations related to hospitalization. Statistical analysis: the data were presented with descriptive statistics.

RESULTS: The final sample consisted of a total of $n=900$ patients, $54 \%$ were male. The mean age was 57.3 years. Of the patients admitted, $192(21.3 \%)$ had a blood glucose value greater than or equal to 140 $\mathrm{mg} / \mathrm{dl}$, and at 72 hours this indicator rose to 486 (54\%) patients. Regarding sex, no differences were found in glucose measurements. Hyperglycemia occurred in a higher proportion in those patients who had an unscheduled hospitalization: $156(81 \%)$ vs. $42(19 \%)$. This finding increased at 72 hours. Of the total number of patients, there were $126(14 \%)$ with a previous history of diabetes. Glycosylated hemoglobin was measured in $426(47 \%)$ patients who had hyperglycemia on admission or at 72 hours, 72 (16.9\%) in glycosylated hemoglobin $>6.5 \%$, who could be defined as patients with diabetes mellitus of recent diagnosis. Glycated hemoglobin $<6.5 \%$ had $342(80.2 \%)$ patients, which could be interpreted clinically as the group of patients with stress hyperglycemia. This group was associated in order of frequency with the following characteristics: postsurgical status, treatment with corticosteroids, active infection, immunosuppressive therapy, acute renal failure, and parenteral nutrition or enteral feeding. Hypertension was the most frequent personal history.

CONCLUSIONS: In this study, the proportion of adults patients with hyperglycemia was 20\%; this proportion is similar to that published in the literature. Glycemic values greater than $140 \mathrm{mg} / \mathrm{dl}$ were present in patients with and without diabetes mellitus; the latter being more frequent among those hospitalized for surgeries and infections, and in those who were receiving treatment with corticosteroids. Of importance, an important number of cases of diabetes mellitus were diagnosed during the hospitalization.

Keywords: inpatient hyperglycemia, stress hyperglycemia.

\section{Introducción}

Las últimas guías internacionales definen hiperglucemia hospitalaria como cualquier valor mayor de $140 \mathrm{mg} / \mathrm{dL}^{1-2}$ al momento de la admisión, en pacientes con y sin antecedentes de diabetes mellitus ${ }^{3}$.

Las causas más frecuentemente encontradas son: estrés metabólico secundario a enfermedad aguda, lo cual produce elevación de hormonas de contra regulación y citoquinas que causan insulinorresistencia; diabetes no diagnosticada; fármacos como corticoides, inmunosupresores, simpaticomiméticos; alimentación parenteral y enteral y reposo prolongado ${ }^{4}$.

Se recomienda el uso de hemoglobina glicosilada (HbA1c) sobre la prueba de tolerancia oral a la glucosa (PTGO) como prueba diagnóstica. La medición de una HbA1c durante los períodos de hospitalización brinda la oportunidad de diferenciar a los pacientes con hiperglucemia por estrés de aquellos con diabetes que no se diagnosticaron previamente ${ }^{5}$. 
La hiperglucemia constituye un factor pronóstico de evolución de la patología durante la internación, aparición de complicaciones e incremento de la mortalidad. Los pacientes con hiperglucemia tienen mayor riesgo de complicaciones intrahospitalarias tales como neumonía, infección de vías urinarias, enfermedad renal, sepsis ${ }^{6}$. Por otra parte, afecta la inmunidad celular y humoral, además de que es un factor procoagulante ${ }^{3}$.

La edad, la hipertensión arterial sistémica, el antecedente de cardiopatía isquémica, la insuficiencia renal aguda, el uso de estatinas son factores que están más frecuentemente asociados a hiperglucemia durante la internación y son predictores independientes de muerte . $^{3}$

La American Diabetes Association y la American Association of Endocrinologists sugiere como óptima una concentración de glucemia entre 140$180 \mathrm{mg} / \mathrm{dL}^{7}$. En el estudio de Umpierrez et al. se encontró que la hiperglucemia estaba presente en el 38\% de los pacientes ingresados en el hospital, y que un tercio de estos pacientes no tenían antecedentes de diabetes antes del ingreso ${ }^{8}$. Wexler et al. demostraron que casi uno de cada cinco pacientes adultos con hiperglucemia de estrés tienen una posible diabetes no conocida, lo que habla del hecho de que casi un tercio de los pacientes con diabetes desconocen su diagnóstico ${ }^{10}$.

\section{Objetivo}

Establecer la frecuencia de hiperglucemia en pacientes internados en la Clínica Universitaria Reina Fabiola.

Secundarios: -Determinar la frecuencia de pacientes con hiperglucemia con antecedente previo de diabetes mellitus.

- Determinar la frecuencia de pacientes diabéticos no conocidos al momento de la internación.

- Determinar la frecuencia de pacientes no diabéticos con hiperglucemia durante la internación y los factores asociados.

\section{Material y métodos}

Estudio observacional, descriptivo, retrospectivo, que comprende desde agosto de 2019 a diciembre de 2019. Población: Pacientes mayores de 18 años de ambos sexos que habían ingresado al Servicio de Clínica Médica de la Clínica Universitaria Reina Fabiola.

Se evaluó la glucemia plasmática al ingreso y la glucemia en ayuno a las 72 horas de internación, igualmente se evaluó la HbA1c a los pacientes hiperglucémicos que no tenían diagnóstico de diabetes previo al ingreso hospitalario. Se consideró hiperglucemia a valores de glucemia mayor o igual a $140 \mathrm{mg} / \mathrm{dl}$, se infirió diagnóstico de diabetes en el momento de la internación a HbA1c mayor o igual a $6.5 \%$ y pacientes con hiperglucemia de estrés a los que tenían HbA1c menor a $6.5 \%$ y alguna causa que lo justifique.

\section{Variables analizadas}

- Valor de glucemia en $\mathrm{mg} / \mathrm{dl}$, medida mediante método hexoquinasa en analizador Cobas 311®, laboratorio Roche.

- Hemoglobina glicosilada en \%, medida por método inmunoturbidimétrico, en analizador Cobas 311®, laboratorio Roche.

$\begin{array}{ll}- & \text { Edad en años } \\ - & \text { Sexo: masculino y femenino } \\ - & \text { Antecedentes personales: } \\ \circ & \text { Diabetes } \\ \circ & \text { Cardiopatía isquémica } \\ \circ & \text { Accidente cerebro vascular } \\ - & \text { Motivo de internación } \\ - & \text { Situaciones relacionadas a la internación: } \\ \circ & \text { Corticoterapia } \\ \circ & \text { Inmunosupresores } \\ \circ & \text { Nutrición parenteral } \\ \circ & \text { Alimentación enteral } \\ \circ & \text { Tratamiento quirúrgico } \\ \circ & \text { Infecciones asociadas } \\ \circ & \text { Insuficiencia renal aguda }\end{array}$

\section{Análisis estadístico}

Con los datos recopilados de las historias clínicas de los pacientes se creó una base de datos de tipo Excel, la que posteriormente se utilizó para los procesamientos estadísticos. Se calcularon las distribuciones de frecuencias absolutas y relativas (\%) para las variables categóricas; y para las variables continuas las medidas de dispersión y posición. Los resultados se presentan en forma de tablas o gráficos según corresponda.

\section{Consideraciones éticas}

Se respetó la Declaración de Helsinki 2013 y la legislación nacional vigente (ANMAT “Guía de buenas prácticas clínicas en Investigación en salud", Ley 9694 de la provincia de Córdoba y la Ley 25.326 de Habeas Data promulgada el 30 de octubre del 2000), se realizará un registro de datos acorde al artículo 8, resguardando los datos personales y el secreto profesional.

En vista de que la sesión de datos recae bajo el Artículo 11, inciso 3.a) para la realización de estudios epidemiológicos este estudio es Categoría 
I de OMS: Estudio sin riesgo. No será necesario consentimiento de los pacientes en el estudio.

\section{Resultados}

La muestra final estuvo conformada por un total de $\mathrm{n}=900$ pacientes, $486(54 \%)$ fueron de sexo masculino.

La edad media (desviación estándar, DE) fue de $57,3(+/-18,6)$ años. Al categorizar la edad en tramos etarios, se pudo observar que los tramos etarios más frecuentes fueron de 41 a 60 años, y de 61 a 80 años, con $31,3 \%$ y $32,7 \%$ respectivamente. De los pacientes ingresados, $192(21.3 \%)$ tenían un valor de glucemia mayor o igual a $140 \mathrm{mg} / \mathrm{dl}$, y a las 72 horas este indicador se elevó a $486(54 \%)$ pacientes (Figura 1).

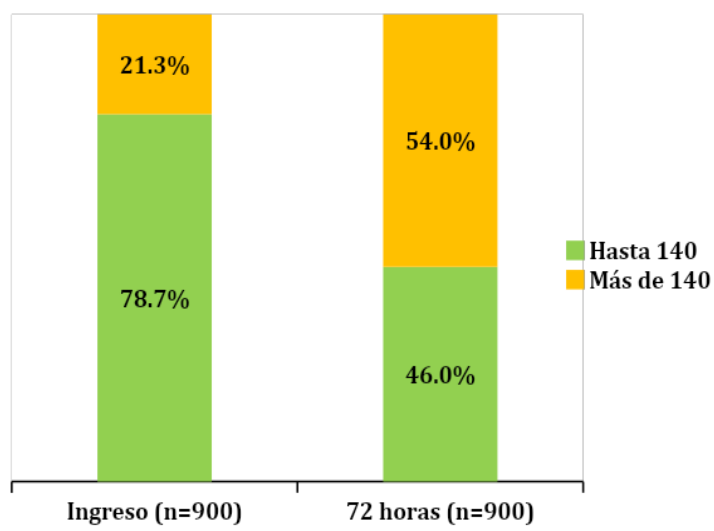

Figura 1. Distribución según Valores de Glucemia y Momento de testeo $(n=900)$.

Los pacientes con glucemia al ingreso mayor a 140 $\mathrm{mg} / \mathrm{dl}$, tendieron a ser mayores que aquellos con glucemias menor a ese valor. Con respecto al sexo, no se hallaron diferencias en las mediciones de la glucosa. Estos datos se muestran en la (Tabla 1).

Tabla 1. Variables demográficas y comorbilidades de acuerdo al valor de la glucemia al ingreso.

\begin{tabular}{|c|c|c|c|}
\hline \multicolumn{2}{|c|}{ Variables } & Glucemia>140 & Glucemia<140 \\
\hline Edad (años) & & $60,3 \pm 20,1$ & $56,4 \pm 18,1$ \\
\hline \multirow{2}{*}{ Sexo (\%) } & Femenino & $43,8 \%$ & $45,8 \%$ \\
\cline { 2 - 4 } & Masculino & $56,3 \%$ & $54,2 \%$ \\
\hline \multirow{4}{*}{$\begin{array}{c}\text { Comorbilidades } \\
(\%)\end{array}$} & Hipertensión & $56,3 \%$ & $34,7 \%$ \\
\cline { 2 - 4 } & Diabetes & $34,4 \%$ & $8,5 \%$ \\
\cline { 2 - 4 } & $\begin{array}{c}\text { Cardiopatía } \\
\text { Isquémica }\end{array}$ & $12,5 \%$ & $4,2 \%$ \\
\cline { 2 - 4 } & ACV & $6,3 \%$ & $5,9 \%$ \\
\hline
\end{tabular}

Al evaluar el motivo de ingreso a la internación, del total de los pacientes con hiperglucemia al ingreso, se presentó en mayor proporción en aquellos pacientes que tuvieron una internación no programada: $156(81 \%)$ vs. 42 (19\%). Este hallazgo en el monitoreo de glucemia a las $72 \mathrm{hs}$ presentó menor diferencia siendo 264 (54.32\%) vs
$222(45.67 \%)$ para no programados y programados respectivamente. (Tabla 2 ).

Tabla 2. Porcentaje de pacientes con hiperglucemia al ingreso y a las 72 horas de acuerdo al tipo de internación.

\begin{tabular}{|l|c|c|}
\hline & $\begin{array}{c}\text { Internación no } \\
\text { programada }\end{array}$ & $\begin{array}{c}\text { Internación } \\
\text { programada }\end{array}$ \\
\hline Hiperglucemia al ingreso & $81 \%$ & $19 \%$ \\
\hline $\begin{array}{l}\text { Hiperglucemia a las 72 } \\
\text { horas }\end{array}$ & $54.32 \%$ & $45.67 \%$ \\
\hline
\end{tabular}

Del total de pacientes, 126 (14\%) tenían diagnóstico previo de diabetes mellitus. De éstos, $66(52,4 \%)$ presentaron valores de glucemia mayores a $140 \mathrm{mg} / \mathrm{dl}$ al ingreso y $84(66,7 \%)$ a las 72 horas (Tabla 3 ).

Tabla 3. Valores de Laboratorio según Antecedente de Diabetes.

\begin{tabular}{|l|c|}
\hline \multirow{2}{*}{ Variables } & Diabetes \\
\cline { 2 - 2 } & Si $(\mathbf{n = 1 2 6})$ \\
\hline Glucemia ingreso $>140$ & $52,4 \%$ \\
\hline Glucemia $72 \mathrm{hs}>140$ & $66,7 \%$ \\
\hline
\end{tabular}

Como se mencionó, se detectaron 192 (21.3\%) pacientes con hiperglucemia al ingreso, 66 (34.3\%) tenían diagnóstico previo de diabetes mellitus, mientras que $126(65.6 \%)$ pacientes no presentaban diagnóstico previo de dicha enfermedad.

A las 72 hs, el número de pacientes con hiperglucemia se elevó a 486 (54\%), de los cuales solo $84(17.2 \%)$ eran diabéticos y el resto 402 $(82.7 \%)$ no lo eran.

Se les midió la hemoglobina glicosilada a 426 (47\%) pacientes que habían presentado hiperglucemia al ingreso o a las $72 \mathrm{hs,} 72(16.9 \%)$ presentaron una hemoglobina glicosilada $>6.5 \%$, a los cuales se los podría definir como pacientes con diabetes mellitus de reciente diagnóstico.

En cuanto a los pacientes sin antecedente de diabetes previo a la internación, con presencia de hiperglucemia, pero con una $\mathrm{HBG}<6.5$, se trataba de un grupo de $342(80.2 \%)$ pacientes en total que se podrían interpretar clínicamente como el grupo de pacientes con hiperglucemia de estrés. Sesenta y seis $(19.29 \%)$ de estos pacientes eran hipertensos, $6(1,7 \%)$ pacientes tenían antecedente de cardiopatía isquémica, y la misma cantidad habían sufrido un accidente cerebrovascular. Las internaciones fueron no programadas en 174 $(50.8 \%)$ pacientes y en $168(49.1 \%)$ sí. Solo 54 (15.7\%) pacientes derivaron de unidades de cuidados intensivos. En cuanto a las características asociadas a la internación, $186(54,3 \%)$ eran pacientes cursando postquirúrgico, $108(31,5 \%)$ 
estaban en tratamiento con corticoides, 90 (26.3\%) pacientes cursaban una infección activa y 36 (10.5\%) pacientes tenían indicada alguna terapia inmunosupresora. En mucha menor proporción había pacientes con insuficiencia renal aguda, y bajo nutrición parenteral o alimentación enteral 12 $(3.5 \%), 6(1.75 \%)$ y $6(1.75 \%)$ respectivamente (Figura 2).

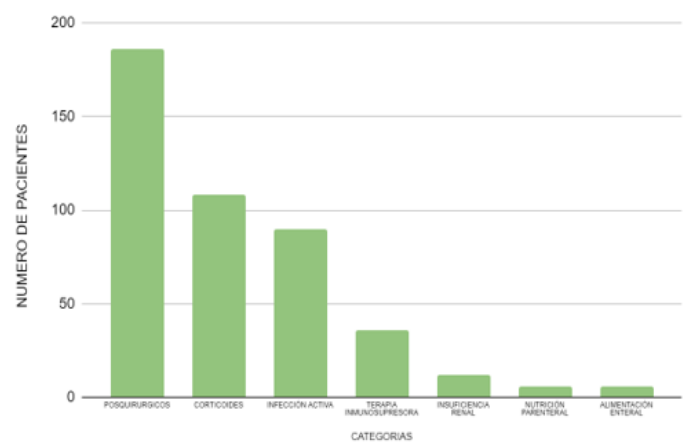

Figura 2. Distribución según Situaciones relacionadas a la internación ( $\mathrm{n}=342)$.

\section{Discusión}

La hiperglucemia es una alteración que tiene implicancias en el pronóstico y la evolución de los pacientes hospitalizados. Es una condición habitual durante la internación, en un estudio se encontró que la hiperglucemia estaba presente en el 38\% de los pacientes ingresados en el hospital 8 , en nuestra muestra se determinó que $21,3 \%$ de los pacientes al ingreso tenían un valor de glucemia superior a $140 \mathrm{mg} / \mathrm{dl}$, y a las 72 horas este indicador se elevó a $54 \%$.

Es muy frecuente la presencia de individuos con diabetes mellitus en la internación, un estudio de cohorte retrospectivo arrojó una prevalencia del $10,99 \%{ }^{9}$, esto coincide con nuestro estudio que tiene un resultado del $14 \%$ de pacientes con esta comorbilidad. El $52,4 \%$ de los pacientes con diabetes ingresaron con hiperglucemia, y este número se elevó a $66,7 \%$ a las 72 hs, pudiendo de esta manera considerar que durante la internación ocurre una descompensación de la enfermedad.

Wexler et al. demostraron que casi uno de cada cinco pacientes con hiperglucemia de estrés tiene probablemente diabetes no conocida identificada por una $\mathrm{HbA} 1 \mathrm{c} \geq 6.5 \%$ lo que habla del hecho de que casi un tercio de los pacientes con diabetes desconocen su diagnóstico ${ }^{10}$. En nuestra muestra 72 pacientes, es decir, uno de cada seis, presentaron este valor en nuestra institución.

Hoy en día se sabe que cualquier enfermedad aguda o cualquier tipo de lesión resulta en resistencia a la insulina, intolerancia a la glucosa e hiperglucemia, una constelación denominada hiperglucemia por estrés o hiperglucemia por lesión aguda ${ }^{4}$. En este estudio, se encontraron 342 pacientes y se pudieron determinar la presencia de algunas causas asociadas, en orden de frecuencia son: estrés postquirúrgico $54,3 \%$, tratamiento con corticoides $31,5 \%$, infección activa $26.3 \%$, terapia inmunosupresora $10.5 \%$ e insuficiencia renal aguda $3.5 \%$.

Los pacientes con apoyo alimenticio excesivo, sobre todo intravenoso, son particularmente propensos a la hiperglucemia ${ }^{4}$, en este trabajo el $3 \%$ de los pacientes con hiperglucemia estaban bajo esta circunstancia. Este valor puede deberse a que solo 48 pacientes del total de la muestra recibieron aporte nutricional, pero de ellos el $25 \%$ desarrolló hiperglucemia.

En general, en pacientes críticos el transporte de glucosa está incrementado 4, en nuestro estudio observamos que el $15 \%$ de los pacientes con hiperglucemia de estrés, derivaron de unidades de cuidados intensivos.

Si bien del total de la muestra la mayoría de los pacientes $(81 \%)$ presentaron hiperglucemia en casos de internaciones no programadas, solo el $15 \%$ de los pacientes con hiperglucemia de estrés pertenecen a este grupo.

\section{Conclusiones}

El porcentaje de pacientes con hiperglucemia en la internación coincidió con la literatura. Valores de glucemia mayores a $140 \mathrm{mg} / \mathrm{dl}$ estuvieron presentes en pacientes con diabetes y en pacientes sin antecedentes de esta enfermedad, es el caso de pacientes que cursaron internación por cirugías e infecciones, y en los que estaban con tratamiento con corticoides. También hubo pacientes en los que con el hallazgo de hiperglucemia se les diagnosticó diabetes durante la internación mediante la medición de la hemoglobina glicosilada.

El relevamiento de los factores asociados a hiperglucemia de estrés, así como la asociación con antecedentes personales, aporta un elemento importante de sospecha de hiperglucemia en nuestro medio y la posibilidad de una intervención terapéutica temprana y efectiva para la prevención de complicaciones que determinan incremento de morbimortalidad de este grupo de pacientes.

\section{Bibliografía}

1. Handelsman Y, Bloomgarden ZT, Grunberger G, Umpierrez G, Zimmerman RS, Bailey TS, et al. American association of clinical endocrinologists and american college of endocrinology - Clinical practice guidelines 
for developing a diabetes mellitus comprehensive care plan - 2015. Endocr Pract. 2015; 21:1-87.

2. American Diabetes Association. 14. Diabetes Care in the Hospital: Standards of Medical Care in Diabetes - 2018. Diabetes Care. 2018; 41: 144-151.

3. Galindo-Garcia G, Galván-Plata ME, NellenHummel H, Almeida-Gutierrez E. Asociación entre hiperglucemia de estrés y complicaciones intrahospitalarias. Rev Med Inst Mex Seguro Soc. 2015; 53: 6-12.

4. Calvo Colindrez JE, Duarte Mote J, Lee Eng Castro VE, Espinosa López RF, Romero Figueroa S, Sánchez Rojas G. Hiperglucemia por estrés. Med Int Mex 2013; 29: 164-170.

5. Farrokhi F, Smiley D, Umpierrez GE. Glycemic control in non-diabetic critically ill patients. Best Practice \& Research Clinical Endocrinology \& Metabolism. 2011; 25: 813824.

6. Moghissi ES, Korytkowski MT, DiNardo M, Einhorn D, Hellman R, Hirsch IB, et al. American Association of Clinical Endocrinologists and American Diabetes Association consensus statement on in patient glycemic control. Diabetes Care. 2009; 32: 1119-1131.
7. Umpierrez GE, Isaacs SD., Bazargan N, Xiangdong YOU, Thaler LM, Kitabchi AE, Hyperglycemia: An Independent Marker of In-Hospital Mortality in Patients with Undiagnosed Diabetes. The Journal of Clinical Endocrinology \& Metabolism. 2002; 87: 978-982.

8. Greci LS, Kailasam M, Malkani S et al. Utility of $\mathrm{HbA}(1 \mathrm{c})$ levels for diabetes case finding in hospitalized patients with hyperglycemia. Diabetes Care 2003; 26: 1064-1068.

9. Russo MP, Elizondo CM, Giunta DH, Grande Ratti MF. Prevalence of hyperglycemia and incidence of stress hyperglycemia in hospitalized patients: A retrospective cohort. Eur J Intern Med. 2017; 43: 15-17.

10. Wexler DJ, Nathan DM, Grant RW, Regan S, Van Leuvan AL, Cagliero E. Prevalence of Elevated Hemoglobin A1c among Patients Admitted to the Hospital without a Diagnosis of Diabetes. J Clin Endocrinol Metab. 2008; 93: 4238-4244.

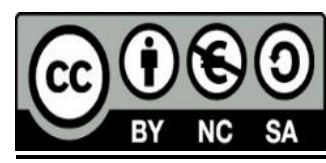

